\title{
Giving ourselves a head start: improving the quality of documentation of referrals to neurosurgery
}

\author{
Madeleine Storey, Simon Webster \\ Cheltenham General Hospital, England
}

\begin{abstract}
Referrals to neurosurgical units are regularly made by doctors in the emergency department (ED), intensive care and acute medicine, following brain injuries sustained by both traumatic and non-traumatic processes. Although some centres accept electronic referrals, many still rely on telephone conversations with a specialist registrar. The flaw in this style of communication is that only information volunteered or requested is relayed. Furthermore, documentation of these dialogues is often incomplete, omitting specific and vital details. Inconsistent advice from referral centres on the management of such brain injury cases had been highlighted, prompting a review of practices at local level in order to improve quality of patient care.
\end{abstract}

The aim of this project was to identify the current level of documentation and improve this through departmental education and implementation of a referral proforma. National guidelines and a literature review were used to formulate the gold standard for high quality documentation.

ED patient notes were retrospectively reviewed over a three month period, assessing adequacy of referral documentation to a neurosurgical centre against the parameters previously set. Initial audit results and specific case studies were presented to ED team members at an educational meeting. A "record of telephone referral to neurosurgery" (RTRN) form was also introduced. Re-audit against the same set of standards was conducted to assess any change in level of documentation and use of the form itself.

The results of this project have shown that, although departmental education improves clinical practice, following the introduction and use of a protocol such as the RTRN there was a significant improvement in the level, and therefore quality of, documentation.

\section{Problem}

This project was carried out at the emergency departments (ED) of Gloucestershire NHS Foundation Trust (GNHSFT), at both Cheltenham General Hospital (CGH) and Gloucester Royal Hospital.

Patients who attend these facilities with symptoms or signs of brain injury may require discussion with specialists at the nearest neurosurgical units. These telephone referrals are often made by middle grade or registrar doctors following confirmation of brain injury on CT imaging.

The problem was exemplified by one 24 hour period at CGH where three patients attended with a Glasgow Coma Scale (GCS) of 3 and were subsequently diagnosed with spontaneous subarachnoid haemorrhage $(\mathrm{SAH})$. All three were promptly referred, but written documentation of the advice received from the neurosurgical centre (as well as referrers and neurosurgical doctors details) were poor. Continued management of such cases is difficult without clear specialist advice, and is further exacerbated by the transfer of the patients between departments and shift changes in staff.

The challenging nature of these cases, where critically unwell patients have a life threatening brain injury, highlighted to ITU and ED staff the potential for improvement not only in patient care and clinical practice but also in open and honest communication with relatives.

\section{Background}

There are sufficient publications surrounding this problem as evidence of existence.[1] What is less well documented is the most appropriate method for improving the quality of documentation following a telephone conversation.

Many neurosurgical centres in the UK now accept e-referrals via a standard online tool. The benefit of this is that it requires all vital details to be present, enabling an informed clinical decision. Where telephone conversations remain the chosen mode of referral, it is highlighted in literature that documentation of advice is often incomplete and recall of the conversation by both parties is poor.[2] The team who noted this developed a pro forma to be sent from neurosurgery back to the referrer in attempt to improve accuracy and prevent criticism.

\section{Baseline measurement}

The Royal College of Physicians guidelines [3] state that every entry in medical notes should include certain pieces of information. Other details deemed to be important in the documentation of referrals to neurosurgery, including what information was given and received, were agreed upon with senior ITU and ED consultants to 


\section{BMJ Quality Improvement Reports}

include the following:

- Date and time of entry

- Name and grade of doctor making referral

- Name and grade of doctor consulted

- Consultant responsible

- Management plan documented clearly

- Vital signs of the patient, including GCS on arrival and best GCS.

These six standards were then used for audit of current levels of documentation.

Measurements were made by retrospective review of three months of patient notes. Electronic ED systems were searched by clinical coding of discharge diagnosis to include head injury, skull fracture, and SAH.

Following baseline analysis, documentation was quantified and poor in most areas. Generally, the name and grade of the doctor making the referral was clear $81 \%$ of the time. However, not one referral documented the information that they had passed on, such as patient vital signs. Further details were documented as shown:

- Date and time of entry: $39 \%$

- Name and grade of doctor consulted: $51.5 \%$

- Consultant responsible: $6 \%$

- Management plan documented clearly: $61 \%$

- Vital signs of patient: $0 \%$.

See supplementary file: ds4142.pdf - "Record of Telephone Referral to Neurosurgery form"

\section{Design}

A documentation proforma was designed in collaboration with senior consultants from ITU and ED for use when making referrals to neurosurgery.

The form was devised as an easy to use intervention with little room for human error, one that would ensure that important information was relayed and the clinical decision regarding patient management easily documented.

This simplicity allows for future documentation to be made more transparent and measurable. Using a paper based tool was more economical than opting into electronic referral systems that are used in other locations, and this reduced cost allowed for quicker implementation. As the form was for use only by Gloucestershire NHS Trust, it did not need approval from other parties.
The form allows the referrer to document all relevant clinical findings before making that phone call, to enable a comprehensive yet succinct account of the patient to be given. There are then areas to document the outcome to reduce ambiguity going forward.

The form will be sustainable as a tool as long as doctors are educated in its existence and encouraged to use it. As staff rotate through positions during their training, it will be highlighted at induction to ensure consistent use.

\section{Strategy}

The initial results following review of patient notes were presented at a departmental teaching session, as well as details on the background of the problem with case studies to emphasise the need for improvement. The RTRN form was also introduced at this time.

Following on from this, two PDSA cycles aided analysis of the implementation and usefulness of the proposed intervention as well as assessment of it's sustainability.

PDSA cycle 1:

The "record of telephone referral to neurosurgery" was introduced simultaneously to the ED departments and intensive care units at both hospitals within GHNHST. After two weeks, feedback was collected regarding the ease and benefits of use. Comments were positive on the content of the form, however there was confusion for some in accessing it. It became apparent that it was available on the intranet but in a different location than expected. Following this feedback, the location of the RTRN form was publicised more widely to improve access and increase use.

PDSA cycle 2:

Following implementation of the pro forma, results of re-audit showed that with it's use, documentation quality vastly improved. However, uptake of the form was only $43 \%$. This highlighted the need for more formal education regarding the existence and availability of the form. Departmental induction to ED was used as the platform to improve this, allowing all newly rotating staff to be made aware of the RTRN as opposed to only staff on shift at departmental handover.

\section{Results}

The initial study identified 69 patients, of which 33 were discussed with neurosurgery. The level of documentation was measured against the parameters set. Of 33 cases, not one documented all of these aspects.

Following the interventions of departmental education, RTRN implementation and confirmation of form location, the level of documentation improved. Re-audit data is shown in numerical form below, with baseline measurements shown again in brackets and first re-audit shown in square brackets. For example: - Result after 


\section{BMJ Quality Improvement Reports}

PDSA 2 [Result after PDSA 1] (Result at baseline). Please see the attached chart which highlights the trend in improvement:

- Name and grade of doctor making referral: $97 \%$ [100\%] (81\%)

- Date and Time of entry: 66\% [62\%] (39\%)

- Name and Grade of doctor consulted: 92\% [90.5\%] (51.5\%)

- Consultant responsible: $31 \%$ [38\%] (6\%)

- Management plan documented clearly: 78\% [76\%] (61\%)

- Vital signs of patient, including pupil reactivity: 100\% [43\%] (0\%).

After PDSA Cycle 1, $43 \%$ of referrals were assisted by use of the form. Following PDSA Cycle 2, where education was more formal as staff began in the department, this increased to $53 \%$. When utilised, documentation was more comprehensive and of greater use to those accepting the patients following stabilisation in ED. For example, with use of the RTRN, five of the six set standards were met $100 \%$ of the time. This serves as evidence that using a pro forma (as well as departmental education) guides one to fill in each section, resulting in increased detail being recorded.

See supplementary file: ds4956.pdf - "Chart showing the improvement in documentation level with the use of education and RTRN implementation 2"

\section{Lessons and limitations}

Educating a department on poor practice is a challenging task. However, it was important to emphasise this as an area for the whole team to address. Requesting a change of practice in staff with set ways was difficult, yet eased by juniors who adapted well and reacted positively to the prospect of an aid to both making a high quality referral and documenting the clinical decision.

The obstacle of where to find the form, highlighted by the request for feedback, was one of practicality and had been initially overlooked when focusing on the content of the RTRN form.

The change in rotation and new staff coming to the department compounded the difficulty with education as an intervention. Education and introduction of the RTRN at future departmental inductions across both hospital sites will ensure its sustainability as an intervention.

\section{Conclusion}

This project has emphasised that, although education improves clinical practice, performance is far superior when documentation is completed using a protocol, such as the "record of telephone referral to neurosurgery". This change will improve patient care and family liaison. Departmental induction is likely to become the forum for increasing awareness of the initial problem, and the effectiveness of the RTRN tool. Going forward, the form would be useful for patients not previously referred (or following new brain injury) on acute medical and other specialty wards.

\section{References}

1. Walters KA. Telephoned head injury referrals: the need to improve the quality of information provided. Arch Emerg Med 1993 Mar;10(1):29-34.

2. Cartmill M, White BD. Telephone advice for neurosurgical referrals. Who assumes duty of care? Br J Neurosurg 2001 Dec;15(6):453-5.

3. RCP Approved 'Generic Medical Record Keeping Standards'. Prepared by the Health Informatics Unit of the Royal College of Physicians. Available at www.rcplondon.ac.uk/resources/structure-and-contentmedical-notes

\section{Declaration of interests}

None declared.

\section{Acknowledgements}

Rob Stacey. 